\title{
The Role and Future Challenges of Wireless Communication Networks for Cooperative Autonomous City Vehicles
}

\author{
Andrei Furda, Laurent Bouraoui, Michel Parent, and Ljubo Vlacic \\ Intelligent Control Systems Laboratory (ICSL) \\ Griffith University, Brisbane, Australia \\ a.furda@griffith.edu.au, 1.vlacic@griffith.edu.au \\ Intitut National de Recherche en Informatique et en Automatique (INRIA) \\ Team IMARA, 78153 Le Chesnay, France \\ laurent.bouraoui@inria.fr, michel.parent@inria.fr
}

\begin{abstract}
This paper elaborates on the use of future wireless communication networks for autonomous city vehicles. After addressing the state of technology, the paper explains the autonomous vehicle control system architecture and the Cybercars- 2 communication framework; it presents experimental tests of communication-based real-time decision making; and discusses potential applications for communication in order to improve the localization and perception abilities of autonomous vehicles in urban environments.
\end{abstract}

Keywords: communication for autonomous city vehicles, Cybercars-2 communication framework, cooperation.

\section{Introduction}

Communication for autonomous city vehicles is crucial for enabling them to perform cooperative driving maneuvers, and important for improving their safety and efficiency. Vehicle-to-Vehicle (V2V) communication allows nearby vehicles to exchange relevant information about their traffic environment (e.g. potential hazards, accidents, etc.), and/or about their driving intentions, even when they are not in line-of-sight (e.g. around corners or at intersections). In addition, Vehicle-to-Infrastructure (V2I) communication can be used to improve the road network efficiency and reduce pollution by informing autonomous vehicles about traffic conditions (e.g. traffic congestions, alternative routes, etc.) and guiding them accordingly.

The remainder of this paper is structured as follows. Section 1.1 elaborates on the state of technology, Section 2 gives an overview of the autonomous vehicle control system and the Cybercars-2 communication framework, Section 3 explains experimental tests, Section 4 elaborates on potentials of future communication networks for autonomous city vehicles, Section 5 explains current limitations of communication networks, and Section 6 concludes this paper. 


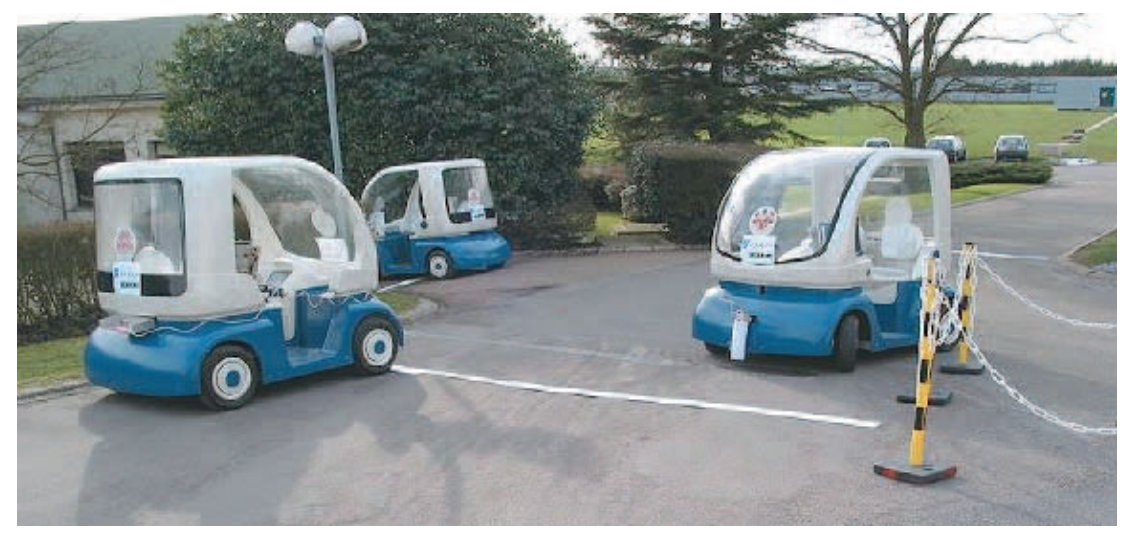

Fig. 1. ICSL/INRIA demonstration of cooperative driving in 2002 [1]

\subsection{State of Technology}

Although the great potentials of using wireless communication for autonomous vehicles are obvious, so far, with the exception of a small number of experimental tests, the numerous ideas about how ad-hoc communication among autonomous vehicles can be used, have not been implemented yet. One of the few early demonstrations were made in 2002, when Griffith University's Intelligent Control Systems Laboratory (ICSL) and INRIA's IMARA Laboratory demonstrated a solution for on-road cooperative autonomous driving. The autonomous vehicles performed driving maneuvers which required inter-vehicle cooperation or synchronization through wireless communication [1]. For example, the vehicles were able to cooperatively overtake each other, and to establish an order of priority before traversing unsignalized intersections (Fig. (1).

Although most recent autonomous vehicle projects do integrate wireless communication functionalities, communication is mainly only used for monitoring the autonomous vehicles and for safety reasons (emergency stopping), and not for enabling cooperative tasks, or for extending the vehicles' input of information regarding the road environment or road infrastructure.

Nevertheless, worldwide standardization efforts addressing the requirements for V2I and V2V communication are currently in progress. One of the most significant efforts is ISO TC204 WG16 and its CALM (Continuous Air Interface for Long and Medium Range) concept. CALM is a set of standards which provides protocols and parameters, specifically addressing the requirements for ITS communication networks 2 .

\section{Autonomous Vehicle Control System Overview and the Cybercars-2 Communication Framework}

\subsection{Autonomous Vehicle Control System}

The ICSL autonomous vehicle control system consists of the following four functional subsystems (Fig. 2): 


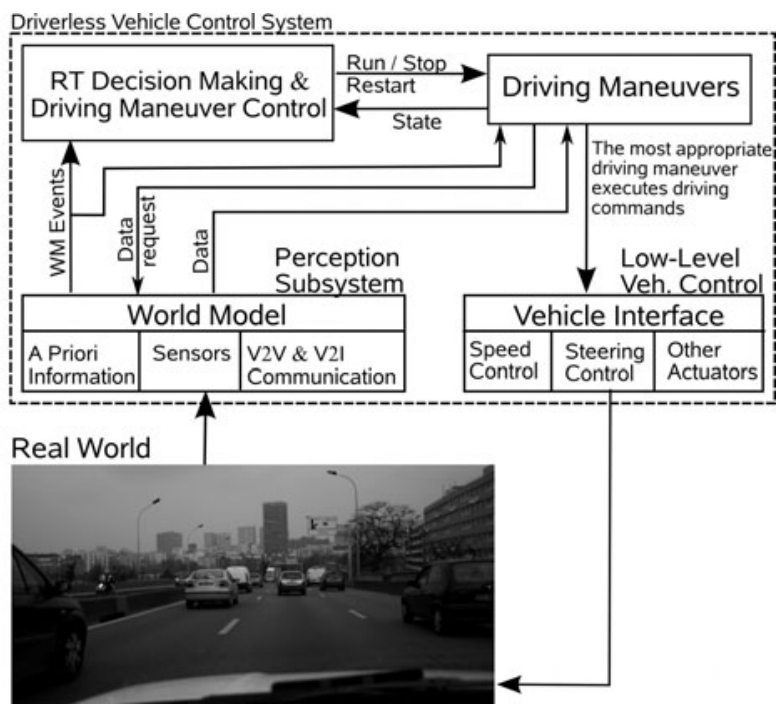

Fig. 2. Autonomous vehicle control system

- Perception Subsystem,

- Real-Time Decision Making \& Driving Maneuver Control,

- Driving Maneuvers,

- Vehicle Interface.

The purpose of the Perception Subsystem is to collect available information about the vehicle's road traffic environment, to manage and process it, and to provide it in an adequate form to the Real-Time Decision Making \& Driving Maneuver Control, and Driving Maneuvers. The Perception Subsystem's components are (Fig. [3):

- A Priori Information: software components providing information available before the vehicle begins its journey.

- Sensor Components: software and hardware components providing information obtained from on-board sensors.

- Communication: software and hardware components providing information obtained through communication with other vehicles (V2V) or infrastructure (V2I) (e.g. traffic management centre).

- World Model (Fig. 33): software component which collects information from subsystems, maintains an up-to-date view of the vehicle's environment, actively notifies other subsystems about relevant events in the traffic environment, and provides access to all its information to other software components through an API (Application Programming Interface).

Based on the information provided by the Perception Subsystem, the Real-Time Decision Making \& Driving Maneuver Control subsystem makes driving decisions. This software subsystem decides about the activation and the execution of the most appropriate driving maneuver for any given traffic situation. 


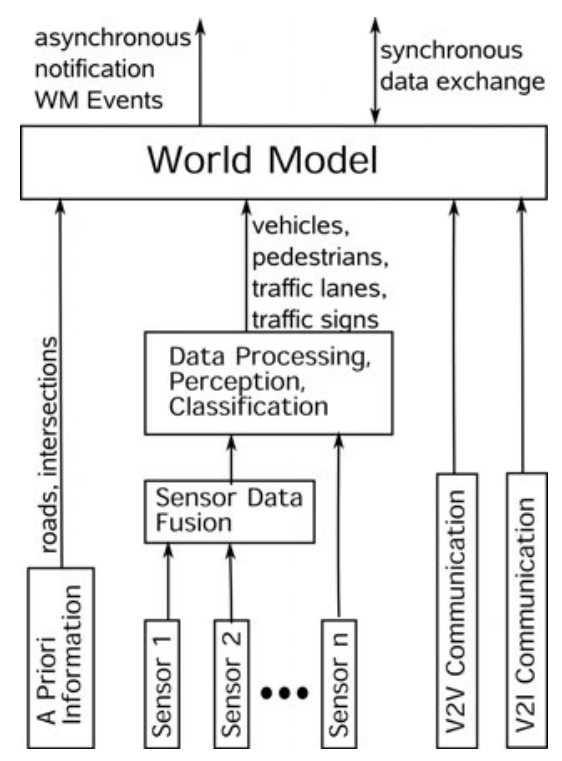

Fig. 3. World Model information input and output

The Driving Maneuvers subsystem contains a set of closed-loop control algorithms, each able to maneuver the vehicle in a specific traffic situation. The driving maneuvers direct their output to the Vehicle Interface subsystem.

The Low-Level Vehicle Control subsystem contains hardware and software components, which control the vehicle's speed, steering angle, and other actuators (e.g. transmission).

The data contained in the World Model is constantly updated in real -time. The main purpose of the World Model within the vehicle control software is to provide the Real -Time Decision Making \& Driving Maneuver Control module, with accurate information about the vehicle's environment. This is accomplished in two ways:

- by actively notifying registered observer modules when the status of World Model Events changed, and

- by allowing other modules to access all information stored in the World Model structure.

The V2V/V2I component of the developed World Model is realized in compliance with the Cybercars-2 communication framework, which is presented in the following subsection.

\subsection{The Cybercars-2 Communication Framework}

The main objective of the Cybercars-2 Communication Framework [2]3 is to enable autonomous vehicles to safely perform cooperative driving maneuvers. 


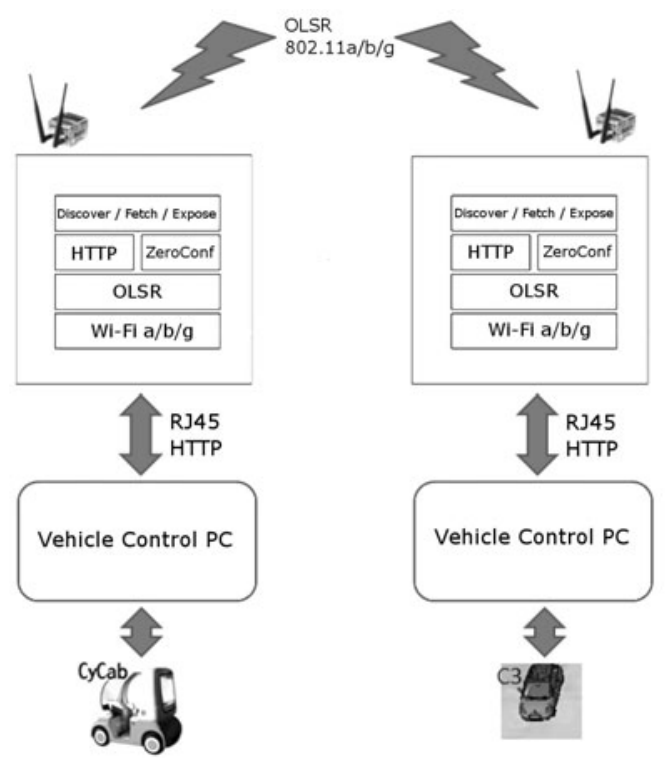

Fig. 4. The Cybercars-2 Communication Framework architecture

Cybercars are Intelligent Transportation Systems 445, based on road vehicles with fully automated driving capabilities. In the current stage, Cybercars are not intended to operate in public traffic, but in restricted environments, such as airports or theme parks.

The communication framework consists of five layers: Physical Layer, MAC Layer, Network Layer, System Service Layer, and Application Layer (Fig. (4).

Physical and MAC Layers: The Cybercars-2 Communication Framework accommodates the use of the following three communication standards / recommendations: IEEE $802.11 \mathrm{p}$, IEEE $802.11 \mathrm{a} / \mathrm{b} / \mathrm{g}$ and WWAN technologies, such as GPRS, UMTS or WiMAX. The IEEE $802.11 \mathrm{p}$ recommendation is used for V2V/V2I communication, IEEE $802.11 \mathrm{a} / \mathrm{b} / \mathrm{g}$ for support information, and WWAN to monitor the traffic flow and to improve its efficiency.

At the current stage, communication equipment compliant to IEEE $802.11 \mathrm{~b} / \mathrm{g}$ is used for V2V and V2I communication. Therefore, the MAC Layer includes the functionalities which are available for the commercial IEEE 802.11 compliant equipment. The hardware and software enabling communication is integrated into the 4G-Cube (Fig. (5)), which is a MIPS-based computer running Linux. It is equipped with one or two Mini-PCI Wi-Fi(b/g) cards and an Ethernet interface [2].

Network Layer: In order to fulfill the main objective, which is to enable cooperative maneuvers, the focus is mainly on close proximity communication between nearby vehicles. In this highly dynamic application environment, involving moving vehicles, dynamic routing is a major requirement. For this purpose, the 

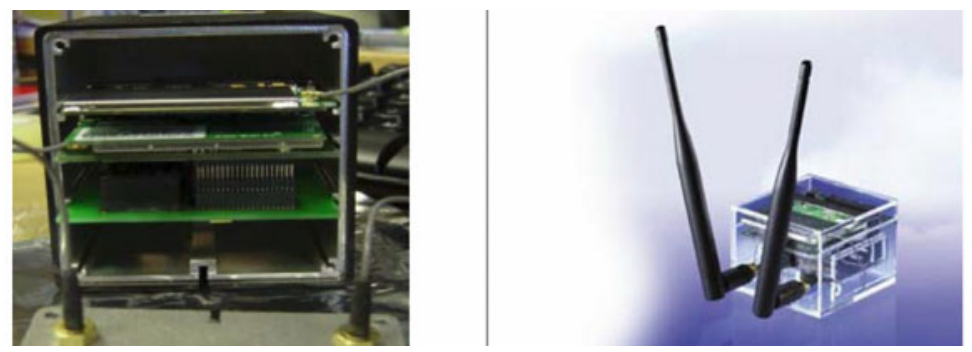

Fig. 5. The 4G-Cube is a small MIPS-based computer with integrated Wi-Fi(b/g) and Ethernet interfaces

Optimized Link State Routing protocol (OLSR) is used. The OLSR protocol provides the functionality required for vehicle communications. It was designed specifically for multihop networks with a strong and efficient mechanism for data flow delivery and it enables the quick creation and reconfiguration of vehicle mesh networks [6]. OLSR operates as a table driven, proactive protocol, allowing the exchange of topology information between network nodes. Each node selects a set of its neighbor nodes as multipoint relays (MPR). Only MPR nodes are responsible for forwarding control traffic, which is intended to be delivered over the entire network. MPRs provide an efficient mechanism to control traffic flooding by optimizing the number of transmissions required, and therefore it helps to avoid network overload.

System Service Layer: As part of the system service layer, the service discovery mechanism Zeroconfiguration from Apple has been adopted, as it helps to improve the network establishment procedure. Multicast DNS (mDNS) is used to provide a local namespace in order to abstract the vehicle's network addresses. On top of DNS (or mDNS), Service Discovery (DNS-SD) can be used by the 4GCube to publish or query information about the applications or services running in the neighborhood [6].

Application Layer: The communication protocol is based on the HTTP 1.1 protocol and uses the HTTP GET and POST requests. The following three functions are provided:

- Discover: to list available services,

- Expose: to send data (i.e. to make data available to all network nodes),

- Fetch: to receive data from a specific network node.

The discover function is used to list all available services. Its main use is for retrieving the list of all communicating vehicles (network nodes) along with the types of information they are able to send. The expose function is used to send communication data. The variety of information sent over the communication network is virtually unlimited. It can include for instance the vehicle's 


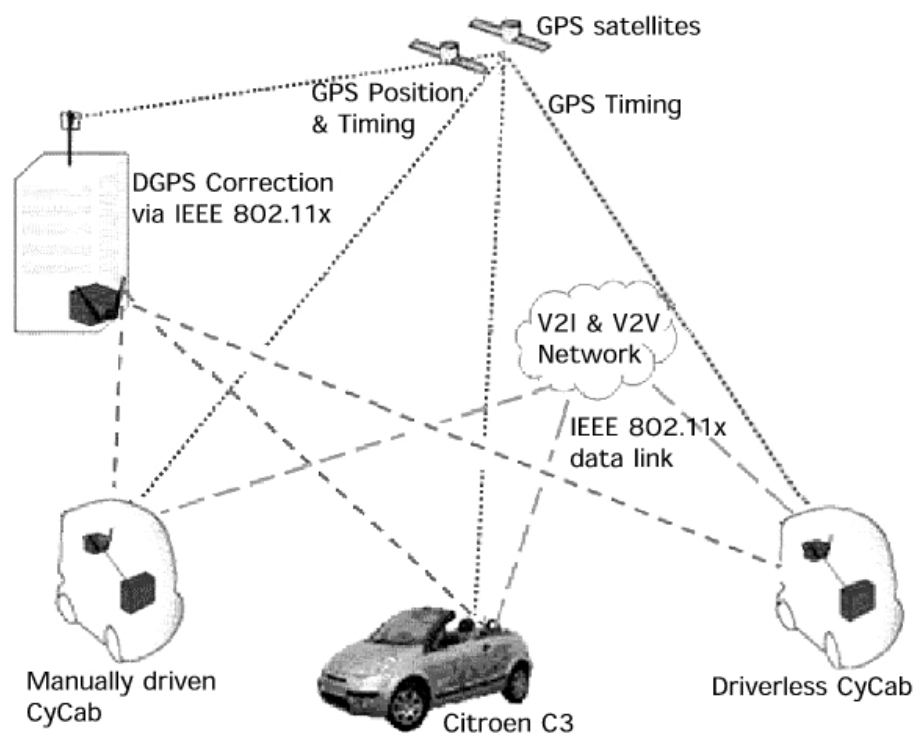

Fig. 6. Overview of the example communication setup (adapted from 2]). The autonomous CyCab communicated with a manually driven CyCab and a conventional car.

current GPS position, speed, heading, data from any on-board sensors, information about its future travel direction, etc. The fetch function is used to receive communication data from a specific network node.

\section{Experimental Decision Making Tests with the Cybercars-2 Communication Framework}

Experimental tests have been carried out in 2009, which show not only the communication performance, but also the potentials of communication for realtime decision making. For these experiments, we used an autonomous vehicle (Cycab, manufactured by Robosoft, France), a second, manually driven Cycab and a conventional car (Citroen C3). All vehicles, sensors, and test facilities haven been provided by the French research institute INRIA (team IMARA), while the decision making approach 7] has been developed at ICSL, Griffith University.

In our experiments, all vehicles, including the conventional car, were equipped with differential GPS (DGPS) and were able to communicate over the communication infrastructure (Fig. 6). In addition to its own GPS position, the autonomous vehicle was able to receive the GPS positions of the other two vehicles. Furthermore, the autonomous vehicle's world model included a priori information, such as the position of intersections and positions of imaginary stop signs. In order to test the decision making approach, three different traffic scenarios 


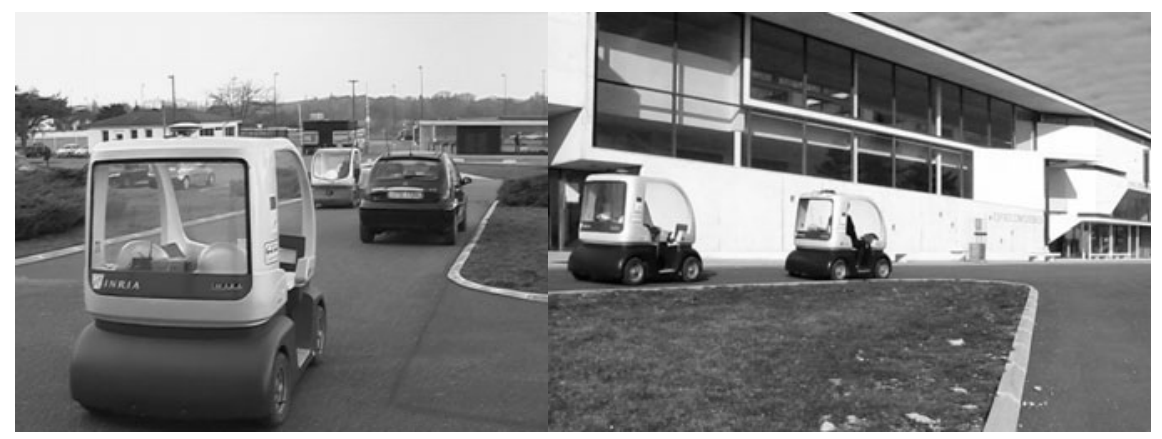

(a) Experiment 2

(b) Experiment 3

Fig. 7. Real-time Decision Making experiments using communication

have been set up, all showing a common decision situation: passing a stopped vehicle under different traffic conditions.

- Experiment 1: In the first traffic scenario, the autonomous vehicle approached a stopped vehicle. Safe passing was possible, and the oncoming traffic lane was free of any obstacles. In this first scenario, the autonomous vehicle immediately started the passing maneuver when it approached the stopped vehicle.

- Experiment 2: The second traffic scenario was similar to the first, however another manually driven vehicle was oncoming, making safe passing impossible (Fig. 7]a). In this second scenario, the autonomous vehicle waited behind the stopped vehicle, and started passing the stopped vehicle when the oncoming traffic lane was free.

- Experiment 3: In the third traffic scenario, a manually driven vehicle was stopped at an intersection (Fig. 7,b). The autonomous vehicle waited behind the stopped vehicle until it crossed the intersection. Then the autonomous vehicle continued driving, stopped at the imaginary stop sign before continuing across the intersection.

The main goal of the experiments related to real-time decision making was to demonstrate that the ICSL autonomous vehicle control software, and most of all the ICSL real-time decision making approach works with real vehicles and real sensors, and meets the real-time decision making requirements. The integration of the Cybercars-2 Communication Framework developed by INRIA into the ICSL control software gave us the first opportunity to test the decision making approach under real-world conditions, while at the same time proving the usability of the Cybercars-2 Communication Framework. During the experimentation phase at INRIA, all experiments were repeated numerous times. Often, new software or hardware related problems were detected and solved. Consequently, these experimental results cannot be regarded as a rigorous test benchmark for the quality of the entire system. 
Although there were a number of remaining problems, such as the unreliable execution of driving maneuvers, and occasional problems related to unreliable DGPS and communication connections, the results show that in all repeated experiments, the decision making module was always able to avoid collisions with other vehicles and make appropriate driving decisions in real-time. Consequently, as real-time decision making was purely based on communication, the results also show that the used communication framework proved to be useful for improving the safe operation of autonomous vehicles.

\section{Future Potentials of V2V and V2I Communication Networks}

\subsection{Vehicle Localization}

One of the major remaining challenges for autonomous city vehicles is the accurate and reliable vehicle localization in urban environments. The currently used Differential GPS (DGPS) technology offers very accurate information, however only when the vehicle is able to receive GPS satellite signals, and, additionally, the position correction signals from a stationary beacon. In urban environments, for example between high buildings or under bridges, the direct reception of satellite signals is not reliable. Therefore, Inertial Navigation Systems (INS) are often used in combination with DGPS, which allow the estimation of position and heading based on inertial measurements and vehicle velocity. However, the accuracy of INS-based estimations decrease within a very short period of time, making such systems useful only for minutes after the GPS satellite reception is lost.

Future communication networks could provide a solution to the localization problem by enabling the vehicles to receive their position on the road from the road infrastructure. Road infrastructure sensors could be used, which detect autonomous vehicles, and inform them about their current position.

Additionally, autonomous vehicles could receive other close-by vehicles' position information which have GPS reception, and, knowing the distance to these vehicles from on-board sensors, calculate their own position.

\subsection{Perception}

Another major challenge is the autonomous vehicle's ability to reliably recognize relevant traffic features, such as traffic signs, intersections, other vehicles, and pedestrians. While currently used LIDAR and RADAR sensors are able to provide very accurate information regarding the distance and velocity of obstacles, they are not able to recognize the type of obstacle. On the other side, in their current state of development, computer vision systems do not seem to provide the required accuracy and reliability in bad weather and light conditions.

As demonstrated in the experimental tests, V2V communication can be used to improve the autonomous vehicles' perception capabilities of communicationenabled vehicles. In the same way, the road infrastructure network could provide relevant information, for example about traffic signs and intersections. 
Since today the majority of people already carry communication devices, such as mobile phones, future networks could use communication with such devices to improve, in addition to vehicle's on-board sensors, the recognition of pedestrians.

\section{Current Limitations and Future Challenges}

\subsection{Current Limitations}

While the currently available wireless communication technology satisfies the needs for mobile non-safety-critical applications such as speech, email, web surfing, entertainment, etc., the currently available wireless networks are not sufficient for safety-critical applications, such as V2V and V2I communication for autonomous vehicles.

The most critical current limitations for wireless V2V and V2I communication networks are:

- Low communication reliability,

- Unsatisfactory network reachability,

- Unsatisfactory real-time performance,

- Inadequate network security.

\subsection{Future Challenges}

Autonomous city vehicles are safety-critical systems. Therefore, if communication is used for purposes which can affect their safe operation, such networks need to guarantee reliability, reachability, fulfillment of real-time requirements, as well as network security requirements.

While occasional communication dropouts are acceptable for non-safetycritical applications, V2V/V2I networks require a very high level of communication reliability. Furthermore, such networks need to guarantee reachability everywhere within the network area, even close to high-voltage power lines, or in tunnels. The worst-case communication times need to be guaranteed within specified real-time limits, regardless of the number of communicating vehicles, amount of transmitted data, or network load.

Network security is another major challenge for future autonomous vehicle communication networks. On such networks, security breaches could have a devastating impact, causing major traffic delays, or, in worst-case scenarios, enable network intruders to take over control over autonomous vehicles.

\section{Conclusion}

This paper has elaborated on the potentials of future wireless communication networks for autonomous city vehicles. After presenting an overview of the autonomous vehicle's control system, the Cybercars-2 communication framework, and experimental tests with wireless communication for real-time decision making, the paper has elaborated on the potentials of V2V and V2I communication 
to help overcome today's biggest challenges for autonomous city vehicles: the vehicle localization and perception. Furthermore, the paper has addressed current limitations and future challenges for future wireless networks for autonomous city vehicles.

\section{References}

1. Kolodko, J., Vlacic, L.: Cooperative Autonomous Driving at the Intelligent Control Systems Laboratory. IEEE Intelligent Systems 18(4), 8-11 (2003)

2. Molinete, B., Bouraui, L., Naranjo, E., Kostense, H., Hendriks, F., Alonso, J., Lobrino, R., Isasi, L.: CyberCars-2: Close Communications for Cooperation between CyberCars. Technical Report Project No IST-2004-0228062 (2009)

3. Bouraoui, L., Parent, M.: Cybercars-2 Close Communications for Cooperation between CyberCars. In: Field Testing Reports, INRIA Technical Report D.4.2 (2006)

4. Parent, M., Gallais, G.: Intelligent transportation in cities with CTS. In: ITS World Congress, Chicago, USA (2002)

5. Parent, M., Gallais, G.: Cybercars: Review of first projects. In: Ninth International Conference on Automated People Movers, Singapore (2003)

6. Clausen, T., Jacquet, P., Laouiti, A., Muhlethaler, P., Qayyum, A., Viennot, L.: Optimized link state routing protocol. In: IEEE INMIC (2001)

7. Furda, A., Vlacic, L.: Towards increased road safety: Real-time decision making for driverless city vehicles. In: 2009 IEEE International Conference on Systems, Man, and Cybernetics, San Antonio, TX, USA (2009) 